\title{
Performance of a molecular assay to detect Mycobacterium tuberculosis complex DNA in clinical specimens: multicenter study in Brazil
}

\author{
Mirela Verza', Karen Barros Schmid ${ }^{1}$, Regina Bones Barcellos ${ }^{1,8}$, Natali Linck \\ Graziele Lima Bello7, Daniel Scapin7, Rosa Dea Sperhacke², Márcia Susana Nunes Silva7, \\ Claudia Wollheim ${ }^{3}$, Martha Gabriela Celle Rivero ${ }^{4}$, Afrânio Lineu Kritski ${ }^{5,8}$, Leonides Rezende Jr ${ }^{6}$, \\ Martha Maria Oliveira ${ }^{5,8}$, Elis Regina Dalla Costa ${ }^{1,8}$, Maria Lucia Rosa Rossetti ${ }^{1,7,8 /+}$
}

\author{
${ }^{1}$ Centro de Desenvolvimento Científico e Tecnológico, Fundação Estadual de Produção e Pesquisa em Saúde, Porto Alegre, RS, Brasil \\ ${ }^{2}$ Universidade de Caxias do Sul, Laboratório de Pesquisa em HIV/AIDS, Caxias do Sul, RS, Brasil \\ ${ }^{3}$ Universidade de Caxias do Sul, Laboratório de Microbiologia Clínica, Caxias do Sul, RS, Brasil \\ ${ }^{4}$ Universidade Federal de São Paulo, Laboratório Especial de Microbiologia Clínica, São Paulo, SP, Brasil \\ ${ }^{5}$ Universidade Federal do Rio de Janeiro, Faculdade de Medicina, Centro de Pesquisa em Tuberculose do Hospital Universitário Clemen- \\ tino Fraga Filho, Laboratório de Genética e Biologia Molecular, Rio de Janeiro, RJ, Brasil \\ ${ }^{6}$ Labtest, Belo Horizonte, MG, Brasil \\ ־Universidade Luterana do Brasil, Canoas, RS, Brasil \\ ${ }^{8}$ Rede Brasileira de Pesquisa em Tuberculose, Rio de Janeiro, RJ, Brasil
}

BACKGROUND In high tuberculosis (TB) burden countries, there are few data on the performance of new molecular commercialised assays developed locally.

OBJECTIVE To evaluate the performance of a new molecular commercialised assay for TB diagnosis (Detect-TB) in three laboratories.

METHODS A total of 302 sputum samples from an equal number of patients with presumptive diagnosis of pulmonary tuberculosis (PTB) were submitted for routine smear microscopy, culture, and Detect-TB assay at three different sites in Brazil (the cities of Caxias do Sul, São Paulo and Canoas).

FINDINGS Seventy four (24.7\%) TB cases were diagnosed (65 bacteriologically confirmed). When compared to smear microscopy/ culture results, the overall sensitivity and specificity of Detect-TB assay was 84.6\% (CI 95\%; 73.7-91.6) and 93.1\% (CI 95\%; 89.195.8), respectively. When compared to bacteriological and clinical diagnostic criteria, the sensitivity and specificity of Detect-TB assay was 74.3\% (CI 95\%; 63.3-82.9) and 92.9\% (CI 95\%; 88.7-95.6), respectively. Among the three sites - Caxias do Sul, São Paulo and Canoas - the sensitivity and specificity were respectively $94.7 \%$ and $97.8 \% ; 71.4 \%$ and $93.9 \%, 82.1 \%$ and $88.9 \%$.

MAIN CONCLUSIONS These findings suggest that the Detect-TB assay could be applied routinely in reference laboratories across different regions in Brazil.

Key words: tuberculosis - reverse-hybridisation assay - IS6110 - molecular diagnosis - Detect-TB

The early and accurate diagnosis of tuberculosis (TB) remains a crucial step in the management and control of TB. The World Health Organization (WHO) estimates that one third of all TB cases remains undiagnosed (WHO 2015). Despite the advance in TB diagnosis, with the introduction of new molecular tests, the difficulties of an early detection of the cases remains a major challenge.

In 2014, the Brazilian Ministry of Health, following WHO recommendations, implemented the molecular semi-automated test Xpert MTB/RIF (Cepheid Inc., Sunnyvale, California) in 94 of the priority cities to control TB, in order to substitute smear microscopy of patients with presumed diagnosis of pulmonary TB (MS/SVS 2015). This assay provides detection of TB and rifampin

doi: 10.1590/0074-02760160196

Financial support: CNPq/INCT 573548/2008-0, FINEP, Labtest and FEPPS.

+ Corresponding author: mrossett@terra.com.br

Received 1 May 2016

Accepted 31 October 2016 resistance directly from untreated sputum in less than $2 \mathrm{~h}$. The implementation of Xpert MTB/RIF in nine countries under programmatic conditions evaluated by Creswel et al. (2014), verified an increase of $30 \%$ to $50 \%$ in the number of confirmed TB cases, but there was no detectable increase in the number of reported cases. Similar results were found in a study performed in Brazil with 14 laboratories in two cities (Durovni et al. 2014). However, this test remains costly for developing nations despite subsidies, as it requires a constant power supply, and involves at least one annual calibration performed by a trained technician.

Other commercial tests, such as AccuProbe (GenProbe, USA) (O'Donnell et al. 2012) and GenoType MTBDRPlus (Hain Life science, Germany) (Maschmann et al. 2013), are reliable, but too expensive for routine use in most low-resource countries. For these reasons, new molecular commercialised assays for TB diagnosis locally developed may be useful and more cost effective.

Our group has previously developed and evaluated an in-house polymerase chain reaction (PCR) using IS6110 as a target and tested three different procedures to detect 
Mycobacterium tuberculosis complex DNA: agarose gel electrophoresis, membrane hybridisation and microwell plate hybridisation (Rossetti et al. 1997, Sperhacke et al. 2004, Verza et al. 2009, Maschmann et al. 2011, Michelon et al. 2011). Based on the microwell plate hybridisation, the in-house procedure has evolved to a molecular kit, the Detect-TB assay that has emerged to cooperate with smear microscopy on TB diagnosis.

The current study describes the performance evaluation of a commercial molecular assay, Detect-TB, developed by LabTest, Belo Horizonte, Brazil, which detects the M. tuberculosis complex in respiratory clinical specimens. Taking into account the different disease incidence rates and laboratory characteristics, we have carried out a multicenter study in three Brazilian cities.

\section{MATERIALS AND METHODS}

Study sites - The multicenter study was carried out in three reference laboratories located in different cities in Brazil from January 2011 to December 2012. The aim was to evaluate the performance of commercial Detect-TB assay compared to reference standard methods. The following laboratories were included: (a) Laboratório Especial de Microbiologia Clínica (LEMC), Universidade Federal de São Paulo (UNIFESP), São Paulo city, in the Southeast Region; (b) Laboratório de Microbiologia Clínica (LMC), Universidade de Caxias do Sul (UCS), Caxias do Sul city, in the South Region; and (c) Laboratório de Biologia Molecular (LBM), Universidade Luterana do Brasil, Canoas city, in the South Region. These sites were chosen for their different incidence rates of 54.2, 22.0 and 94.2 of TB per 100,000 inhabitants, respectively. LEMC-UNIFESP is a routine and research laboratory, and evaluates clinical samples from patients admitted to the University Hospital in São Paulo, LMC-UCS is a reference laboratory for clinical evaluations in Caxias do Sul, and LBM-ULBRA is a research laboratory that analyses clinical samples from Health Units in Canoas city.

Specimens and patients - 302 sputum samples (one sample per patient), on the basis of routine testing, from presumed pulmonary TB patients without primary TB treatment in the past, were collected to estimate the accuracy of the Detect-TB assay: 42 clinical specimens from São Paulo, 112 from Caxias do Sul, and 148 from Canoas. Mycobacterial cultures and smear microscopy were carried out for all clinical samples. Bias was minimised through blinding in which the technicians performing the molecular and reference tests were unaware of the other test results.

The sputum specimens were treated with ZiehlNeelsen staining and culture on Löwenstein-Jensen medium and identification of $M$. tuberculosis complex (MTBC) with biochemical tests, according to MS/SVS recommendations (MS/SVS 2008).

Procedure - The method, based on Michelon et al. (2011), was developed by our group at the Centro de Desenvolvimento Científico e Tecnológico (CDCT), Fundação Estadual de Produção e Pesquisa em Saúde (FEPPS), Porto Alegre, Brazil, and patented at INPI (Instituto Nacional da Propriedade Industrial) under number PI 0900612-5. It began to be commercialised after registration at ANVISA with number 10009010259, named Detect-TB assay.
Briefly, M. tuberculosis (MTB)-complex DNA was amplified using biotinylated primers targeting the IS6110 fragment. These amplified products were reverse-hybridised on microwell plates (Nunc Immobilizer ${ }^{\mathrm{TM}}$ Amino Surface, Nunc A/S, Roskilde, Denmark) to a fixed aminated probe complementary to the internal region of the amplified IS6110 fragment. The hybridisation signal was detected by colorimetry using the streptavidinperoxidase/TMB system and measured using a spectophotometer with a 450/620 nm filter. The absorbance of the negative control was subtracted from the results and all samples with readings above 0.275 were considered positive for MTB complex DNA, as recommended by Detect-TB (Michelon et al. 2011).

Study design - To evaluate the assay at different rates of TB incidence and geographical locations, Detect-TB assays were sent to the three study sites cited above. All laboratory personnel involved in the study received a detailed protocol and underwent a one-week training course. To facilitate the reproducibility of the results, all tests were performed with commercialised assays from the same production lot, thereby ensuring that the tests used contained reagents from batches that had been produced previously and tested in accordance to the current Brazilian specific law (good manufacturing practices for the control of diagnostic products - ANVISA- decree 686, 08.27.1998).

Bacteriologically confirmed pulmonary tuberculosis (PTB) cases were defined when the patient matched at least one of two of the following criteria: (a) two smear positive for acid-fast bacilli; or (b) M. tuberculosis (Mtb) culturepositive in respiratory specimens. Non-bacteriologically confirmed PTB cases were defined as those that had clinical and radiological diagnosis and clinical improvement after six months of solely anti-TB treatment. Non-TB cases were defined as those that did not receive anti-TB treatment for the following two years, according to the Brazilian National Disease Reporting System (SINAN).

Statistical analysis - Detect-TB assay performance was compared to reference standard methods: TB cases with bacteriologically confirmed criteria and those with composite criteria that include TB cases with and without bacteriologically confirmation. Sensitivity, specificity, accuracy and kappa tests were calculated using the Statistical Package for Social Sciences (SPSS) v16.0. P-values lower than 0.05 were considered statistically significant.

Ethics - This study was approved by the Ethical Committee of Fundação Estadual de Produção e Pesquisa em Saúde do Rio Grande do Sul (FEPPS-RS) under the number $01 / 2007$.

\section{RESULTS}

The cut-off value of the assay $(0.250)$ was defined previously by Michelon et al. (2011). The gray zone was defined as the values from 0.250 to 0.275 . Overall, among 302 clinical samples, only $3(0.99 \%)$ had results in the gray zone. All samples in the gray zone were repeated and the results remained inconclusive. For this reason, these samples were removed from the analysis.

A total of 299 sputum samples were evaluated, 74 (24.7\%) TB cases were diagnosed of which 65 were bacte- 
riologically confirmed (Table). When compared to bacteriological confirmed diagnosis, the overall sensitivity and specificity of Detect-TB assay was $84.6 \%$ (CI 95\%; 73.791.6) and 93.1\% (CI 95\%; 89.1-95.8), respectively. When compared to composite diagnosis criteria, the sensitivity and specificity of Detect-TB assay was $74.3 \%$ (CI 95\%; 63.3-82.9) and 92.9\% (CI 95\%; 88.7-95.6), respectively. Median turnaround time from sputum collection to the release of Detect-TB assay results was two days.

In Canoas city, 39 of the 148 analysed samples had positive results using smear microscopy or culture methods. Detect-TB assay showed a sensitivity of $82.1 \%$ (CI $95 \% ; 67.0-91.0$ ), specificity of $88.9 \%$ (CI 95\%; 81.8-93.7), and a kappa value of 0.682 (CI 95\%; 0.611-0.766) (Table). According to composite diagnosis criteria, of the 148 samples from Canoas, 41 had positive results. Detect-TB assay sensitivity, specificity kappa value were of $78.1 \%$ (CI 95\%; 63.1-88.2), 90.5\% (CI 95\%; 83.2-94.9) and of 0.654 (CI 95\%; 0.573-0.734), respectively (Table).

At the Caxias do Sul site, 19 of the 112 samples had positive results using smear microscopy and/or culture methods. Detect-TB assay showed sensitivity of $94.7 \%$ (CI 95\%; 90.0-99.3), specificity of 97.8\% (CI 95\%; 94.6100.0), and a kappa value of 0.907 (CI 95\%; 0.848-0.965) (Table). According to composite diagnostic criteria, 20 of the 112 samples tested were positive for TB. DetectTB assay showed sensitivity of $90.0 \%$ (CI 95\%; 83.996.0 ), specificity of $97.8 \%$ (CI 95\%; 94.6-100.0), and a kappa value of 0.878 (CI 95\%; 0.812-0.943) (Table).

In São Paulo city, seven of the 42 analysed samples had positive results using smear microscopy or culture methods. Detect-TB assay showed sensitivity of $71.4 \%$ (CI 95\%; 56.1-86.6), specificity of 93.9\% (CI 95\%; 85.2102.0), and a kappa value of 0.654 (CI 95\%; 0.494-0.813) (Table). According to composite diagnostic criteria, 13 out of 42 samples at the São Paulo site tested positive. Detect-TB assay showed a sensitivity of 38.5\% (CI 95\%; 22.1-54.8), specificity of $92.6 \%$ (CI 95\%; 83.2-101.0), and a kappa value of 0.353 (CI 95\%; 0.192-0.513) (Table).

\section{DISCUSSION}

A multicenter study was carried out for the performance evaluation of the Detect-TB assay for the detection of M. tuberculosis complex DNA in clinical samples. For detection, amplified products with the expected size of $245 \mathrm{bp}$ (IS6110) were reverse-hybridised to a microwell plate. This test was selected for its simple method and colorimetric end point, factors that facilitate the adoption of new nucleic acid amplification tests at laboratories at least in middle income countries that can provide laboratories with molecular techniques (Dowdy et al. 2003, van Cleeff et al. 2005).

At the three sites (Canoas, Caxias do Sul and São Paulo cities), when compared to confirmed bacteriological diagnosis, the overall sensitivity of the DetectTB assay ranged from 82.1 to $97.8 \%$ and the specificity ranged from $71.4 \%$ to $94.7 \%$. When compared to composite diagnostic criteria, sensitivity ranged from $38.5 \%$ to $90.0 \%$ and specificity ranged from $88.7 \%$ to $97.8 \%$. The results obtained at these sites are in agreement with the findings from other studies and with commercialised assays (Piersimoni \& Scarparo 2003, Ling et al. 2008, Michelon et al. 2011, O'Donnell et al. 2012, Bhutia et al. 2013, Steingart et al. 2013). The rate of false-positive results in all sites were of $2.2 \%$ at Caxias do Sul, $6.9 \%$ at Sao Paulo and $8.1 \%$ at Canoas.

Detect-TB is an assay to be performed in research and/or clinical laboratories according to the manufacturer's instructions. As any other molecular technique, good laboratory practices must be followed, including unidirectional workflow, different rooms for specimen separation and processing, and reagent preparation. The use of a negative control in all steps of the protocol, from DNA extraction to DNA amplification detection, ensures the identification of contamination event during the analysis. However, this handling procedure occurs at post sample separation. The higher rate of false positive results observed in Canoas city (8.1\%) compared to the other sites could be explained due to cross-contamination that could have occurred during specimen processing. Although we followed all the guidelines for diagnostic methods, before the molecular analysis, at the Hospital Laboratory, the samples may have been manipulated in the same cabinet that the solid culture was performed, and this could have led to sputum contamination, which can only be detected at the molecular level. Generally, the results of Detect-TB assay showed better specificity with composite diagnostic criteria when compared to laboratory criteria. In a previous study by our group, we demonstrated that smear microscopy plus DetectTB assay was the most cost-effective initial diagnostic test for active TB when applied in a prison population with a high prevalence of active PTB, indicating that Detect-TB assay has great potential to be implemented under routine diagnostic conditions (Schmid et al. 2014). The overall rate of false negatives was higher when the Detect-TB assay was to composite diagnostic criteria (6.3\%) than with bacteriological criteria (3.3\%). A high number of false-negative samples was found in São Paulo $(19.0 \%$ with clinical diagnosis and $4.7 \%$ with laboratory diagnosis). This observation is most likely related to the characteristics of the TB patients evaluated in a university hospital, which is a tertiary care unit that is not the reference for TB treatment. The patients assisted at this level of care are usually more paucibacillary, as reported by others (Michelon et al. 2011). The incorrect processing of the samples during homogenisation and decontamination could also have led to unequal aliquots during processing for smear microscopy, culture and PCR. This phenomenon may have resulted in an insufficient number of detectable bacilli that were suitable only for detection by smear or culture. In this case, an inappropriate sample dilution during the decontamination procedure or a sampling error could have reduced the sensitivity of the test, generating a false-negative result as described elsewhere (Woods 2001, Piersimoni \& Scarparo 2003, Chakravorty et al. 2005). The DetectTB assay has proved sensitive to the detection of smear microscopy negative samples (Michelon et al. 2011). In this study, Detect-TB assay was able to detect four cases of smear microscopy positive with culture negative samples, probably due to the presence of non-viable bacte- 


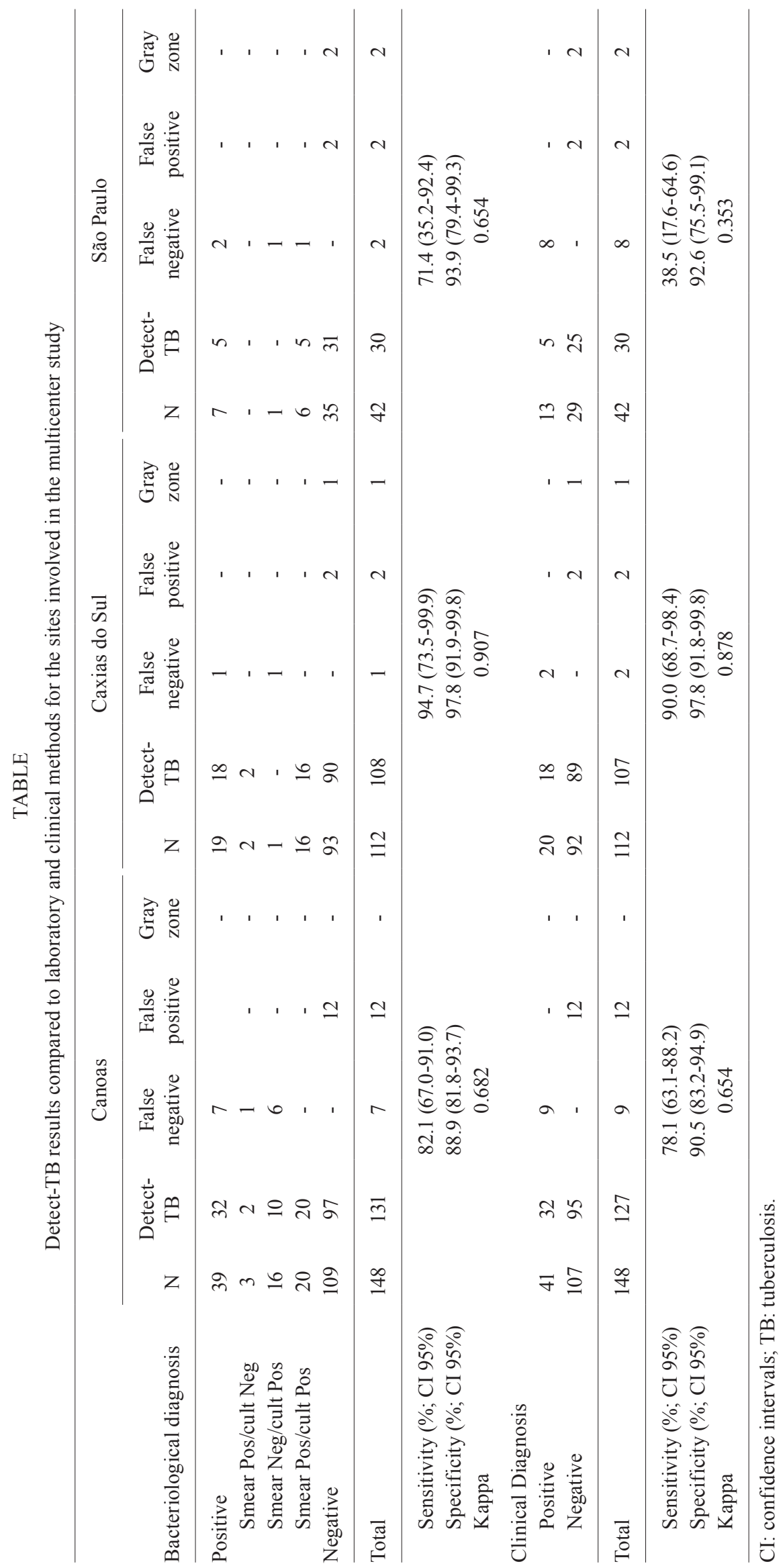


ria, and notably detected 10 cases out of 16 of smear microscopy negative with culture positive samples. In this scenario, Detect-TB assay results were released in only two days, lower than the turnaround time related to the culture results ( $\sim 30$ days). Depending on the prevalence of active TB in the population, nucleic acid amplification taken along with the full clinical picture could be useful to rull in or rull out the anti-TB treatment. The nucleic acid amplification test result is only one piece of evidence and would not alone define a clinical decision. One limitation of our study might rely on the assumption that we considered as TB cases those with two positive smear microscopy and culture negative. More than one sputum positive smear microscopy result supports the diagnosis of mycobacterial respiratory disease but does not allow differentiation of TB from non-mycobacterial disease. Additionally, it is uncommon to have non-mycobacterial diseases with two positive smear microscopy. In our sample it occurred in only three cases. Therefore, we can infer that those cases had a high probability to be active TB cases instead a non-mycobacterial disease.

There is a high degree of variability across studies in the accuracy of nucleic acid amplification tests. Assuming the TB prevalence at each site and the accuracy based upon the final clinical criteria plus laboratory confirmed diagnosis of PTB observed at the three different laboratories (Table), high negative predictive values $(94 \%$ in Canoas, $89 \%$ in Caxias and 95\% in São Paulo) and moderate positive predictive values ( $71 \%$ in Canoas, $89 \%$ in Caxias and $70 \%$ in São Paulo) were obtained for all sites. Those figures are also achieved even with Xpert MTB/ RIF. Assuming that Xpert MTB/RIF has a sensitivity of $90 \%$ and specificity of $98 \%$, having HIV negative presumed TB subjects evaluated at health services with TB prevalence of $1 \%$ at primary health care centers, $10 \%$ at emergency units and $20 \%$ at hospitals or reference ambulatory centers, the negative predictive values achieved with this test were respectively $99.8 \%, 98.8 \%$ and $97.5 \%$, and positive predictive values $31.0 \%, 83.0 \%, 92.0 \%$. Smear microscopy and culture techniques remain important diagnostic techniques to be used in addition to Xpert MTB/RIF, in regions with low TB prevalence, in order to increase the positive predictive value.

Therefore, molecular tests cannot replace culture but should be used in addition to conventional tests and clinical data for TB diagnosis as highlighted by others (Pai et al. 2003, 2004, Piersimoni \& Scarparo 2003, CDC 2009). Furthermore, the Detect-TB assay is similar to an ELISA as it requires common laboratory equipment to reference laboratories from middle income countries with a high burden of TB and HIV, and it has the advantage of being a low test cost (US\$10,00), when compared to other current molecular commercialised assays. In addition, the assay results, which are based on spectrophotometry, are independent of human interpretation, instead of visual interpretation colorimetric assays (Kox et al. 1996).

\section{AUTHORS' CONTRIBUTION}

MV conducted the study, conducted the analysis of laboratory tests, participated in data acquisition, performed the statistical analysis, and drafted of the manuscript; KBS, RBB and
ERDC participated in laboratory tests, performed data analysis, participated in data acquisition, and drafted the manuscript; MGCR and CW participated in laboratory tests and participated in data acquisition; MLRR participated in laboratory tests, participated data analysis and helped draft the manuscript; RDS, participated data analysis, participated in the acquisition of data, and helped draft the manuscript; GLB, NL and DS participated in laboratory tests; LRJ helped design the study, participated data analysis and helped draft the manuscript; MSNS participated data analysis and drafted the manuscript; AK conceived the study, participated in its design, performed data analysis, drafted the manuscript; $\mathrm{MMO}$ conceived the study, participated in its design, coordinated the study, helped draft the manuscript. All authors contributed to the interpretation of the results and have read and approved the final manuscript.

\section{REFERENCES}

Bhutia R, Narain K, Devi KR, Singh TS, Mahanta J. Direct and early detection of Mycobacterium tuberculosis complex and rifampicin resistance from sputum smears. Int J Tuberc Lung Dis. 2013; 17(2): 258-61.

CDC - Centers for Disease Control and Prevention. Updated guidelines for the use of nucleic acid amplification tests in the diagnosis of tuberculosis. MMWR Morb Mortal Wkly Rep. 2009; 58(1): 7-10.

Chakravorty S, Sen MK, Tyagi JS. Diagnosis of extrapulmonary tuberculosis by smear, culture and PCR using universal sample processing technology. J Clin Microbiol. 2005; 43(9): 4357-62.

Creswell J, Codlin AJ, Andre E, Micek MA, Bedru A, Carter EJ, et al. Results from early programmatic implementation of Xpert MTB/ RIF testing in nine countries. BMC Infect Dis. 2014; 14: 2.

Dowdy DW, Maters A, Parrish N, Beyrer C, Dorman SE. Cost-effectiveness analysis of the gen-probe amplified Mycobacterium tuberculosis direct test as used routinely on smear-positive respiratory specimens. J Clin Microbiol. 2003; 41(3): 948-53.

Durovni B, Saraceni V, van den Hof S, Trajman A, Cordeiro-Santos $\mathrm{M}$, Cavalcante $\mathrm{S}$, et al. Impact of replacing smear microscopy with Xpert MTB/RIF for diagnosing tuberculosis in Brazil: a steppedwedge cluster-randomized trial. PLoS Med. 2014; 11(12): e1001766.

Kox LF, Noordhoek GT, Kunakorn M, Mulder S, Sterrenburg M, Kolk AH. Microwell hybridization assay for detection of PCR products from Mycobacterium tuberculosis complex and the recombinant Mycobacterium smegmatis strain 1008 used as an internal control. J Clin Microbiol. 1996; 34(9): 2117-20.

Ling DI, Flores LL, Riley LW, Pai M. Commercial nucleic-acid amplification tests for diagnosis of pulmonary tuberculosis in respiratory specimens: meta-analysis and meta-regression. PLoS ONE. 2008; 3(2): e1536.

Maschmann RA, Verza M, Silva MSN, Sperhacke RD, Ribeiro MO, Suffys PN, et al. Detection of rifampin-resistant genotypes in Mycobacterium tuberculosis by reverse hybridization assay. Mem Inst Oswaldo Cruz. 2011; 106(2): 139-45.

Maschmann RD, Spies FS, Nunes LD, Ribeiro AW, Machado TR, Zaha A, et al. Performance of the GenoType(R) MTBDRplus assay directly on sputum specimens from Brazilian patients with tuberculosis treatment failure or relapse. J Clin Microbiol. 2013; 51(5): 1606-8.

Michelon CT, Rosso F, Schmid KB, Sperhacke RD, Oliveira MM, Kritski AL, et al. Colorimetric microwell plate reverse-hybridization assay for Mycobacterium tuberculosis detection. Mem Inst Oswaldo Cruz. 2011; 106(2): 194-9.

MS/SVS - Ministério da saúde/Secretaria de Vigilância em Saúde. Detectar, tratar e curar: desafios e estratégias brasileiras frente à tuberculose [Internet]. 2015 [cited 2016 October 31]. Available from: 
http://portalsaude.saude.gov.br/images/pdf/2015/marco/27/2015007---BE-Tuberculose---para-substitui----o-no-site.pdf.

MS/SVS - Ministério da Saúde/Secretaria de Vigilância em Saúde. Manual nacional de vigilância laboratorial da tuberculose e outras micobactérias [Internet]. 2008 [cited 2016 October 31]. Available from: http://bvsms.saude.gov.br/bvs/publicacoes/manual_ vigilancia_laboratorial_tuberculose.pdf.

O'Donnell N, Corcoran D, Lucey B, Barrett. A molecular-based mycobacterial identification in a clinical laboratory setting: a comparison of two methods. Br J Biomed Sci. 2012; 69(4): 164-8.

Pai M, Flores LL, Hubbard A, Riley LW, Colford Jr JM. Nucleic acid amplification tests in the diagnosis of tuberculous pleuritis: a systematic review and meta-analysis. BMC Infect Dis. 2004; 4: 6.

Pai M, Flores LL, Pai N, Hubbard A, Riley LW, Colford Jr JM. Diagnostic accuracy of nucleic acid amplification tests for tuberculous meningitis: a systematic review and meta-analysis. Lancet Infect Dis. 2003; 3(10): 633-43.

Piersimoni C, Scarparo C. Relevance of commercial amplification methods for direct detection of Mycobacterium tuberculosis complex in clinical samples. J Clin Microbiol. 2003; 41(12): 5355-65.

Rossetti MLR, Jardim SB, Rodrigues VFS, Moura AR, Oliveira H, Zaha A. Improvement of Mycobacterium detection in clinical samples using DNA purified by glass matrix. J Microb Methods. 1997; 28(2): 139-46.
Schmid KB, Scherer L, Barcellos RB, Kuhleis D, Prestes IV, Steffen $\mathrm{RE}$, et al. Smear plus detect-TB for a sensitive diagnosis of pulmonary tuberculosis: a cost-effectiveness analysis in an incarcerated population. BMC Infect Dis. 2014; 14: 678.

Sperhacke RD, Mello FCQ, Zaha A, Kritski AL, Rossetti MLR. Detection of Mycobacterium tuberculosis by a polymerase chain reaction colorimetric dot-blot assay. J Tuberc Lung Dis. 2004; 8(3): 312-7.

Steingart KR, Sohn H, Schiller I, Kloda LA, Boehme CC, Pai M, et al. Xpert ${ }^{\circledR}$ MTB/RIF assay for pulmonary tuberculosis and rifampicin resistance in adults. Cochrane Database Syst Rev. 2013; 31(1): CD009593.

van Cleeff M, Kivihya-Ndugga L, Githui W, Ng'ang'a L, Kibuga D, Odhiambo J, et al. Cost-effectiveness of polymerase chain reaction versus Ziehl-Neelsen smear microscopy for diagnosis of tuberculosis in Kenya. Int J Tuberc Lung Dis. 2005; 9(8): 877-83.

Verza M, Maschmann RA, Silva MSN, Costa ERD, Ribeiro MO, Rosso F, et al. In house colorimetric reverse hybridisation assay for detection of the mutation most frequently associated with resistance to isoniazid in Mycobacterium tuberculosis. Mem Inst Oswaldo Cruz. 2009; 104(5): 710-4.

WHO - World Health Organization. Global tuberculosis report 2014 [Internet]. 2015 [cited 2016 October 31]. Available from: http://apps. who.int/iris/bitstream/10665/137094/1/9789241564809 eng.pdf.

Woods GL. Molecular techniques in mycobacterial detection. Arch Pathol Lab Med. 2001; 125(1): 122-6. 